\title{
Anabases
}

ANABASES Traditions et réceptions de l'Antiquité

$17 \mid 2013$

Varia

\section{Chrystèle BLONDEAU, Marie JACOB (éd.), L'Antiquité entre Moyen Âge et Renaissance}

Florence Bouchet

\section{OpenEdition}

Journals

Édition électronique

URL : http://journals.openedition.org/anabases/4253

DOI : $10.4000 /$ anabases.4253

ISSN : 2256-9421

Éditeur

E.R.A.S.M.E.

Édition imprimée

Date de publication : 1 mars 2013

Pagination : 274-276

ISSN : 1774-4296

\section{Référence électronique}

Florence Bouchet, "Chrystèle blondeAu, Marie JacoB (éd.), L'Antiquité entre Moyen Âge et Renaissance », Anabases [En ligne], 17 | 2013, mis en ligne le 01 avril 2013, consulté le 22 septembre 2020. URL: http://journals.openedition.org/anabases/4253 ; DOI : https://doi.org/10.4000/anabases.4253

Ce document a été généré automatiquement le 22 septembre 2020.

(c) Anabases 


\title{
Chrystèle BLONDEAU, Marie JACOB (éd.), L'Antiquité entre Moyen Âge et Renaissance
}

\author{
Florence Bouchet
}

\section{RÉFÉRENCE}

Chrystèle BLONDEAU, Marie JACOB (éd.), L'Antiquité entre Moyen Âge et Renaissance, Nanterre, Presses universitaires de Paris Ouest, 2011, 405 p. +27 pl. en couleurs 25 euros / ISBN 978-2-84016-057-1.

1 Ce recueil constitue les actes du colloque international éponyme (sous-titré «L'Antiquité dans les livres produits au nord des Alpes entre 1350 et 1520 ») organisé les 8,9 et 10 mars 2006 à l'INHA par le centre de recherches Histoire de l'art et représentations (HAR) et l'école doctorale Milieux, cultures et sociétés du passé et du présent de l'université Paris Ouest-Nanterre. Il rassemble 18 articles d'historiens, historiens de l'art et littéraires qui, chacun à leur manière, constatent la vitalité du référent antique dans la culture de la fin du Moyen Âge, bien que le champ de l'enquête, se concentrant sur le royaume de France et les États bourguignons, exclue l'Italie.

2 La première partie (articles de F. Duval, A. Van Buren, S. Hériché-Pradeau, É. Karagiannis-Mazeaud, F. Lemerle) s'attache à «définir l'Antiquité ». Le vocabulaire est un puissant révélateur : contrairement au français moderne, où "Antiquité » et " antique » se réfèrent à une période historique définie, le sème principal de antiquitas ou antiquus, en latin médiéval, est " passé », " ancien », sans autre précision. En ancien et moyen français, "anciens» (adjectif et substantif pluriel) désigne divers types d'autorités issus de la Bible ou de l'héritage gréco--romain, et «temps anciens » ne renvoie pas nécessairement à l'Antiquité, manifestant par là « une conception relative du passé [...] alors que la périodisation de l'historiographie moderne est absolue» 
(p. 43). Cette dernière ne se diffusera qu'à partir du Quattrocento. L'évolution de la représentation du costume romain dans l'enluminure corrobore une prise en compte tardive de sa spécificité. Jusque dans la première moitié $d u \mathrm{xv}^{\mathrm{e}}$ siècle, une forme de «modernisme » assimilateur pousse à figurer les Romains vêtus à la mode médiévale ; au milieu $d u \mathrm{Xv}^{\mathrm{e}}$, les costumes deviennent plus exotiques et manifestent à la fin du siècle un souci archéologique encouragé par l'humanisme. C'est cette même aptitude à reconnaître l'altérité de l'Antiquité que révèle la comparaison de deux versions de l'histoire d'Alexandre le Grand. Alors que Jehan Wauquelin, en 1448, privilégie le merveilleux et la tradition courtoise, Vasque de Lucène, en 1468, rationalise l'approche du conquérant macédonien, tient compte de la dimension païenne et s'efforce de restituer l'épaisseur temporelle de l'Antiquité. Son œuvre est ainsi placée "sous le signe de l'Autre ", quand celle de son prédécesseur l'était "sous le signe du Même " (selon les catégories de Paul Ricœur). En 1512, les Illustrations de Gaule et Singularitez de Troye de Jean Lemaire de Belges jettent un regard ambivalent sur la Grèce. D'un côté, l'auteur garde la marque d'un mishellénisme (attesté de Virgile à Pétrarque) qui lui permet d'affirmer la primauté culturelle de la Gaule; de l'autre, il ne peut échapper à l'intérêt humaniste de son temps pour l'Antiquité. En s'attachant à définir le territoire grec d'un point de vue géopolitique, sans négliger les enseignements de la langue et des mythes grecs (dans une perspective d'acculturation), il a finalement contribué à la vulgarisation de nombreux sujets et concepts helléniques. Le regard porté sur les monuments antiques a lui aussi évolué à la Renaissance. Le Moyen Âge a été essentiellement assimilateur (témoin, le recyclage de vestiges antiques dans les constructions romanes). C'est à la fin $\mathrm{du} \mathrm{xv}^{\mathrm{e}}$ siècle que les antiquités gallo--romaines furent considérées pour elles-mêmes, d'abord par des étrangers (voyageurs des pays germaniques, marchands, diplomates et architectes italiens). Les descriptions qu'ils ont laissées offrent les prémices d'une archéologie de l'Antique, avant que les antiquaires lyonnais et dauphinois ne s'en préoccupent dans la première moitié du XvI ${ }^{\mathrm{e}}$ siècle.

3 La deuxième partie (articles de M.-H. Tesnière, J. Drobinsky, I. Villela-Petit, K. Straub, V. Auclair) s'interroge sur « la transmission des modèles classiques ». La transmission des classiques au Moyen Âge est d'abord affaire de translatio, de traduction. Ainsi celle, en 1358, par Bersuire, d'une partie de l'Histoire romaine de Tite-Live à la demande de Jean II le Bon: le roi pouvait y trouver des exemples utiles pour gouverner. Certains termes de la traduction (regent, prevost) et des gloses glissées dans le lexique qui accompagne le texte favorisent l'actualisation du legs romain. La notoriété d'Ovide, étudié, traduit et commenté tout au long du Moyen Âge, a assuré la pérennité de tout un corpus d'histoires fameuses, telle celle de Héro et Léandre. Dans le lointain sillage des Héroïdes, l'auteur de l'Ovide moralisé, Guillaume de Machaut et Christine de Pizan ont élaboré leur version (parfois plusieurs) de la légende tragique, mettant en valeur tantôt l'action de Léandre, tantôt celle de Héro. À son tour l'enluminure s'empare de la légende et en privilégie, d'un manuscrit à un autre, des détails variables. La fascination des médiévaux pour la mythologie, que la pensée chrétienne saura s'approprier par la doctrine de l'integumentum, a également perpétué dans l'imaginaire des histoires antiques, là encore souvent mises en images. Le dossier sur les travaux d'Hercule fait apparaître des variations dans les exploits retenus par divers auteurs des $\mathrm{XIV}^{\mathrm{e}}-\mathrm{XV}^{\mathrm{e}}$ siècles à partir de la Consolation de philosophie de Boèce, dont la tradition iconographique est examinée. Sous le règne troublé de Charles VI, en plein marasme de la guerre de Cent Ans, le personnage d'Hercule pouvait démontrer qu'il est possible de triompher des épreuves de la vie. Quant à l'héritage de la rhétorique antique, il s'est 
perpétué tout au long du Moyen Âge, comme en témoignent en 1463 Les Douze Dames de rhétorique. Cette œuvre dessine un idéal éthique du poète fondé sur l'idée que l'éloquence a partie liée avec la sagesse et mobilise un art de la mémoire qui est un autre héritage antique. Ainsi se trouve réactualisé le modèle du vir bonus dicendi peritus. Les canons de l'architecture antique, eux, ont connu une reviviscence à la Renaissance. Dès 1505, Jean Pélerin (dit Viator) a écrit le De artificiali perspectiva, premier traité sur la perspective imprimé en France. Il s'approprie les principes définis par Alberti dans le De re Aedificatoria (1485), lui-même nourri du traité d'architecture de Vitruve ( $\mathrm{I}^{\mathrm{er}}$ siècle). Les dessins procurés par Jean Pélerin ont diffusé l'esthétique antique que l'architecture renaissante devait réintégrer à ses propres constructions.

4 La troisième partie (articles de M. Pérez-Simon, M. Madureira, C. Rabel, J.C. Mühlethaler, T. Sol, P. Aladjidi, M.Jacob, R.-M. Ferré) propose des «lectures et interprétations de l'Antiquité ». La lecture des ouvrages païens antiques a d'abord, on le sait, posé un problème idéologique et moral au public chrétien du Moyen Âge. Quoique ce problème ait été tôt surmonté, certains auteurs médiévaux continuent de manifester une gêne face aux récits des Anciens. C'est ce que montre - en creux - le prologue de la vie romancée d'Alexandre le Grand qu'est l'Historia de Preliis (version J 3), dans son effort pour justifier, à la fin du XIII ${ }^{\mathrm{e}}$ ou au début du XIve siècle, la lecture des textes païens, tant par les clercs que par les laïcs. De fait, l'Antiquité offrait aux médiévaux un «stock» de personnages, historiques ou légendaires, et de situations porteurs d'une valeur exemplaire. Mais celle-ci, loin d'être figée, a pu connaître des retournements plus ou moins radicaux au fil des réemplois. Les réécritures faites au xiv siècle du mythe du Jugement de Pâris (Ovide moralisé, Fontaine amoureuse de Guillaume de Machaut, Espinette amoureuse de Froissart) font varier les arguments des trois déesses et la valeur de Pâris, parfait amant, traître ou fou porte-malheur. Le philosophe stoïcien Sénèque, à la charnière de l'âge antique et de l'âge chrétien, devint au Moyen Âge une auctoritas de premier plan et fut parfois même considéré comme un cryptochrétien (on lui prêta une correspondance apocryphe avec l'apôtre Paul). L'iconographie le concernant privilégie sa mort (qui confine au martyre), son statut d'enseignant et d'autorité morale. Le sort de Palinure, le nautonier d'Énée, quoique moins célèbre, se prête aussi à une série de réinterprétations variables en fonction des détails soulignés. Victime expiatoire nécessaire dans l'Énéide, Palinure se charge d'ambiguïté jusqu'à devenir "un guide sur le chemin de la perdition» (p.318), comme en témoigne en particulier le double traitement du personnage chez Octovien de Saint-Gelais (Séjour d'Honneur, Eneydes). Dans une perspective politique, Jules César lui-même devient un tyran démagogue mais au fond coupé de la communauté des hommes selon Nicole Oresme qui traduisit et commenta la Politique d'Aristote pour Charles V en 1372. À cette époque, en effet, l'universalisme impérial était en contradiction avec la volonté de la monarchie française de légitimer son pouvoir sur un territoire identifié - et limité - à la nation. La malléabilité des exemples antiques se vérifie aussi d'un point de vue moral dans les miroirs des princes aux XIV et $\mathrm{xV}^{\mathrm{e}}$ siècles. La libéralité d'Alexandre le Grand et de Titus, qui pourrait a priori constituer une vertu à imiter, est relue de façon critique comme inadéquate à l'esprit de charité qui doit présider à l'aumône chrétienne ; en contrepoint, Trajan, quoique d'abord persécuteur de chrétiens, a su par ailleurs rendre la justice d'une façon que louent Philippe de Mézières et Christine de Pizan. Il faut attendre la seconde moitié $\mathrm{du} \mathrm{Xv} \mathrm{e}^{\mathrm{e}}$ siècle pour que certains auteurs se déprennent de la vision du Gaulois barbare et sanguinaire transmise par Justin, Tite-Live ou César ; les miniatures des manuscrits du Romuleon de Sébastien Mamerot témoignent de cette 
évolution qui tend à réhabiliter un peuple que les Français étaient en train de se reconnaître pour ancêtre. Ainsi, l'Antiquité peut aussi bien offrir des modèles prestigieux que des contre-exemples, ou encore se charger d'un esthétisme plus ou moins exotique. Ces différentes veines apparaissent dans les spectacles organisés à la cour de René d'Anjou entre 1446 et 1478.

5 Au total, ce volume réorchestre des problématiques désormais assez bien identifiées grâce aux nombreux travaux consacrés à la réception de l'Antiquité au Moyen Âge, notamment depuis les deux dernières décennies. Le champ d'enquête est immense ; l'intérêt de ce volume - malgré un titre un peu plat - est donc d'ouvrir de nouveaux dossiers invitant à lire ou relire des textes parfois peu connus et à observer des images inédites jalousement protégées par les bibliothèques. Nul doute que les analyses proposées susciteront d'autres prolongements.

\section{AUTEURS}

\section{FLORENCE BOUCHET}

Université de Toulouse (UTM)

bouchet@univ-tlse2.fr 